\title{
Non-coding genomic regions possessing enhancer and silencer potential are associated with healthy aging and exceptional survival
}

\author{
Sangkyu Kim ${ }^{1}$, David A. Welsh ${ }^{2}$, Leann Myers ${ }^{3}$, Katie E. Cherry ${ }^{4}$, Jennifer \\ Wyckoff ${ }^{1}$, S. Michal Jazwinski ${ }^{1}$ \\ ${ }^{1}$ Tulane Center for Aging and Department of Medicine, Tulane University Health Sciences Center, New Orleans, LA 70112, \\ USA \\ ${ }^{2}$ Department of Medicine, Louisiana State University Health Sciences Center, New Orleans, LA 70112, USA \\ ${ }^{3}$ Department of Biostatistics and Bioinformatics, School of Public Health and Tropical Medicine, Tulane University Health \\ Sciences Center, New Orleans, LA 70112, USA \\ ${ }^{4}$ Department of Psychology, Louisiana State University, Baton Rouge, LA 70803, USA \\ Correspondence to: \\ Sangkyu Kim, e-mail: skim5@tulane.edu
}

Keywords: aging, frailty, longevity, linkage, association, non-coding

Received: November 11, $2014 \quad$ Accepted: December 8, 2014

Published: February 28, 2015

\section{ABSTRACT}

We have completed a genome-wide linkage scan for healthy aging using data collected from a family study, followed by fine-mapping by association in a separate population, the first such attempt reported. The family cohort consisted of parents of age $\mathbf{9 0}$ or above and their children ranging in age from 50 to 80 . As a quantitative measure of healthy aging, we used a frailty index, called FI $_{34}$, based on 34 health and function variables. The linkage scan found a single significant linkage peak on chromosome 12. Using an independent cohort of unrelated nonagenarians, we carried out a fine-scale association mapping of the region suggestive of linkage and identified three sites associated with healthy aging. These healthy-aging sites (HASs) are located in intergenic regions at 12q13-14. HAS-1 has been previously associated with multiple diseases, and an enhancer was recently mapped and experimentally validated within the site. HAS-2 is a previously uncharacterized site possessing genomic features suggestive of enhancer activity. HAS-3 contains features associated with Polycomb repression. The HASs also contain variants associated with exceptional longevity, based on a separate analysis. Our results provide insight into functional genomic networks involving non-coding regulatory elements that are involved in healthy aging and longevity.

\section{INTRODUCTION}

Aging can be defined as the occurrence of changes over time that adversely affect the vitality and functions, increasing the mortality rate [1]. The onset of aging varies from individual to individual, and the agingrelated changes occur at different rates in different individuals at many levels of biological organization. This complex phenomenon has both genetic and non-genetic underpinnings $[2,3]$. The finding that lifespans of various model organisms can be altered, sometimes dramatically, by single gene mutations suggests a role for genes in aging $[3,4]$. On the other hand, near doubling of human life expectancy in developed countries during the past two centuries attests to the complexity of the etiology as well as the importance of environmental factors [5].

One quantitative indicator of the genetic basis of a complex trait is heritability, which is a measure of the extent of genetic control of the trait. The heritability of human longevity ranges from 0.15 to $0.35[6,7]$, which also implies that $65-85 \%$ of the trait can be controlled by non-genetic factors. However, studies indicate that survival to older ages is under stronger genetic influence. For example, siblings of centenarians are four times more likely to survive to their early nineties compared with siblings of 73-year olds, and siblings of centenarians are at 
least 8 to 17 times more likely to reach age 100 compared with their birth cohort controls $[8,9]$. Healthy, long-lived people are likely to carry more beneficial genetic variants, fewer harmful variants or both.

Efforts to find such genetic elements have been made using genetic epidemiological methods [10]. A major hurdle in genome-wide studies of complex diseases is the lack of sufficient statistical power. The lack of power in complex trait studies comes from inadequate setting of a number of important statistical parameters, such as significance level, sample size, and effect size [11, 12]. One approach to alleviate the power demand is to base an experiment on the prior odds generated from the preceding study [11]. A paradigm of this approach is to carry out genome-wide linkage analysis and fine-scaled association mapping of linkage regions [13, 14]. Accordingly, we set up the Healthy Aging Family Study (HAFS) for linkage analysis [15]. Our plan was to dissect linkage-defined regions with association mapping, using our ongoing association studies [16].

For a quantitative measure of heathy aging, we developed the frailty index $\mathrm{FI}_{34}$, composed of 34 common health and function variables [15]. $\mathrm{FI}_{34}$ increases exponentially with age, indicating declining health and function ability. The rates of increase differ significantly between offspring of long-lived parents ( $\geq 90$ years old) and offspring of short-lived parents $(<76$ years old at death), indicating that $\mathrm{FI}_{34}$ is associated with parental longevity. The genetic basis of $\mathrm{FI}_{34}$ is substantiated by a narrow-sense heritability estimate of 0.39 . Using $\mathrm{FI}_{34}$, we found elevated levels of resting metabolic rate (RMR) linked to declining health in nonagenarians [17]. This result points to RMR as an important physiological marker of healthy aging among the oldest-old. It also illustrates the use of $\mathrm{FI}_{34}$ as a means of identifying additional physiological factors involved in healthy aging.

We have completed genome-wide linkage scanning followed by fine-scale association mapping and found three sites associated with healthy aging at 12q13-14. These HASs are located in intergenic regions. Functional annotation of the HASs indicates that they possess genomic features indicative of enhancer or silencer activity. Our results indicate that healthy aging, and longevity as well, can be controlled by non-coding regulatory elements.

\section{RESULTS}

\section{A single linkage peak for healthy aging at 12q13-14}

When the non-parametric linkage $(\mathrm{npl})$ analysis in the MERLIN package was carried out on the data from the HAFS offspring only, the most significant linkage peak was found at $77 \mathrm{cM}\left(\mathrm{LOD}=2.3, P=6.0 \times 10^{-4}\right)$ on chromosome 12 (Figure 1A). Because the healthy aging phenotype data were unavailable for HAFS parents, we inferred healthy aging status of each parent from the parent-offspring linear regression, where the slope of the regression line is mathematically equivalent to the narrow-sense heritability of $\mathrm{FI}_{34}$ (see METHODS). When the inferred parental data were incorporated in the $\mathrm{npl}$ analysis, the linkage peak on chromosome 12 became more significant with a higher LOD score (Figure 1B; LOD = 3.0, $P=1.0 \times 10^{-4}$ ). Dense SNP markers can result in inflation of linkage when significant linkage disequilibrium (LD) between adjacent markers exists (see METHODS). However, linkage analysis software assumes markermarker linkage equilibrium. Therefore, it is important to take into account potential marker-marker LD in linkage analysis. We obtained similar linkage results with different models of LD between SNP markers (Figure S1). Thus, the $n p l$ analysis delineated a region suggestive of linkage to healthy aging on chromosome 12 . The SNPs showing LOD of 3.0 lie in a region of about $1 \mathrm{Mb}$ in size.

\section{Three healthy-aging associated sites (HAS)}

For fine-scale mapping, we genotyped 175 LHAS nonagenarians for whom $\mathrm{FI}_{34}$ data were available, and applied linear regressions to genotypes of 330 SNPs that are located within the linkage peak (LOD > 2.7). The dependent variable was the $\mathrm{FI}_{34}$ score and the independent variable was the genotype of the SNP marker in the additive mode. Age and sex were included as covariates. This way, we tested whether the risk of healthy aging or unhealthy aging among the oldest-old increases additively as the number of copies of the minor allele increases, after adjustment for age and sex. Three groups of SNPs stood out in this analysis (Figure 2A): SNPs in HAS-1 (the lowest $P=3.5 \times 10^{-3}$ ), SNPs in HAS-2 (the lowest $P=4.2$ $\times 10^{-3}$ ), and SNPs in HAS-3 (the lowest $P=1.3 \times 10^{-2}$ ). Employing the very conservative Bonferroni correction for multiple comparisons to maintain a family-wise error rate of 0.05 , no SNP exceeded the adjusted significance level of $1.52 \times 10^{-4}$ (Linear regression in Table 1).

Because the linkage outcome was based on the binary coding of $\mathrm{FI}_{34}$, we dichotomized $\mathrm{FI}_{34}$ as the binary status of healthy versus unhealthy aging (see METHODS) and applied logistic regression to the same SNPs with age and sex as covariates. This time, SNPs in HAS-2 and HAS- 3 remained conspicuous, whereas HAS-1 became less prominent (Figure 2B). The top SNP in HAS-2 $\left(P=2.0 \times 10^{-4}\right)$ was very close to the significance cutoff set by the Bonferroni adjustment (Logistic regression in Table 1). HAS-1, -2 , and -3 , delineated by the top four SNPs in each, are $\sim 40 \mathrm{~kb}, \sim 340 \mathrm{~kb}$, and $\sim 250 \mathrm{~kb}$ long, respectively. SNPs in HAS-1 were previously associated with various diseases [18], whereas the SNPs in HAS-2 and HAS-3 have not been associated with any phenotypes before. 

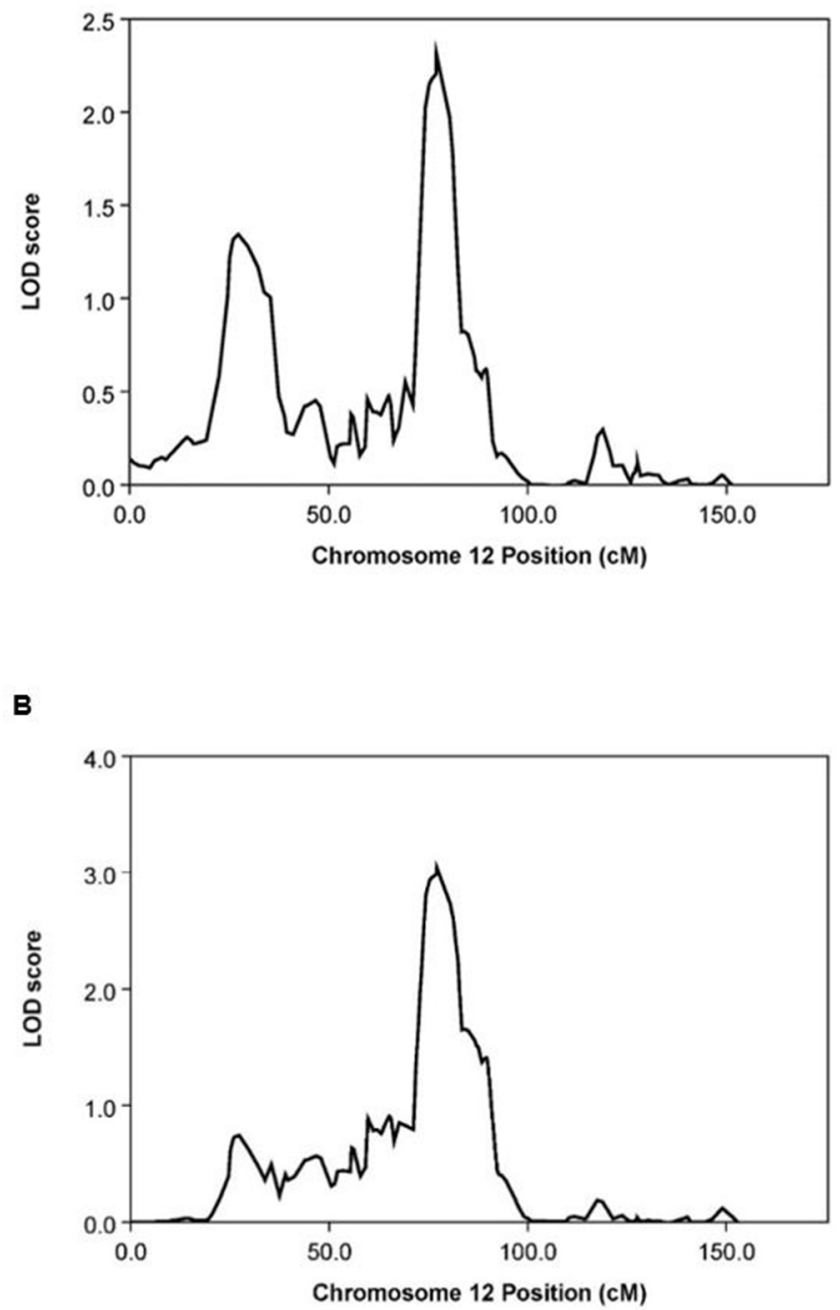

Figure 1: Graphical summary of MERLIN $\boldsymbol{n p l}$ analysis on chromosome 12. (A) From offspring data only (LOD $=2.3$, $P=6.0 \times 10^{-4}$ ). (B) From offspring data combined with inferred parental phenotype data (LOD = 3.0, $P=1.0 \times 10^{-4}$ ).

We also examined association of the same set of SNPs with longevity. For this, we genotyped LHAS controls of ages from 39 to 59 and compared allele frequencies between these controls and the nonagenarian cases (Figure 2C). Compared with healthy-aging associated SNPs, more SNPs were associated with longevity from among the 330 chromosome 12q13-14 SNPs genotyped, and many of these associations were highly significant. Also, the longevity-associated SNPs were not limited to the HASs.

\section{Genomic features of HASs}

We examined the genomic features of the healthyaging associated sites using computational annotation tools. As shown previously [18], a number of proteincoding genes exist in HAS-1, and promoter regions are marked with higher levels of epigenetically modified histones and DNase I sensitive sites compared to non-promoter regions (Figure S2). In addition, the promoters contain transcription factor binding sites that are co-located with chromatin segments indicative of strong enhancer activity. Unlike HAS-1, HAS-2 is largely devoid of any protein-coding genes (Figure S3). However, it contains several features indicative of regulatory elements, such as clusters of H3K4Me1 and $\mathrm{H} 3 \mathrm{~K} 27 \mathrm{Ac}$ histone marks, DNase I sensitive sites, and transcription factor binding sites. The absence of elevated levels of $\mathrm{H} 3 \mathrm{~K} 4 \mathrm{Me} 3$, which is usually found near promoters, is consistent with the absence of promoters in HAS-2. All these features coincide with the presence of several strong enhancers across multiple cell lines. In stark contrast with HAS-1 and HAS-2, HAS-3 lacks such active chromatin features (Figure S4). Instead, HAS-3 has multiple Polycomb-repressed blocks in multiple cell lines and a locus encoding a long intergenic noncoding RNA (lincRNA). The full length of this RNA is about $71 \mathrm{~kb}$. 

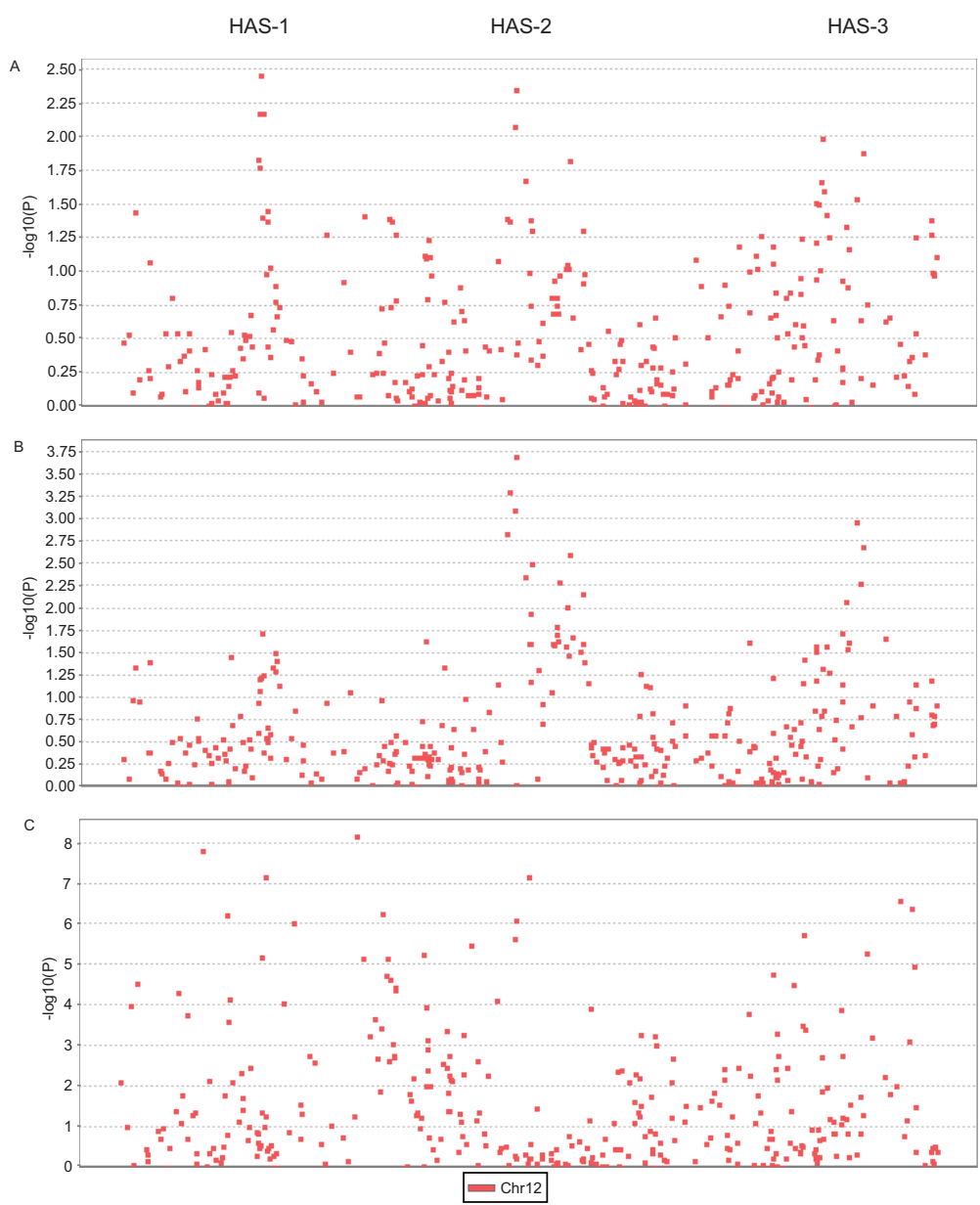

Figure 2: Manhattan plots of association results. (A) $-\log _{10} P$ values from linear regressions of $\mathrm{FI}_{34}$ scores on additive effects of 330 SNPs, with sex and age differences adjusted, were plotted against SNP positions. Applying a Bonferroni adjustment, the cutoff significance $P$ value is $1.52 \times 10^{-4}$ and its $-\log _{10}$ counterpart is 3.8. (B) The same as in (A) but using logistic regressions of dichotomized $\mathrm{FI}_{34}$ values. $\left(\right.$ C) $-\log _{10}$ $P$ values from $\chi^{2}$ tests for differences in allele frequencies between oldest-old cases and young controls were plotted against SNP positions.

Table 1: SNPs associated with healthy aging at 12q13-14

\begin{tabular}{|c|c|c|c|c|c|c|}
\hline \multirow[b]{2}{*}{ HAS } & \multirow[b]{2}{*}{ SNP } & \multirow[b]{2}{*}{ Position ${ }^{\dagger}$} & \multicolumn{2}{|c|}{ Linear regression } & \multicolumn{2}{|c|}{ Logistic regression } \\
\hline & & & Coefficient & $P$ & OR & $P$ \\
\hline \multirow{4}{*}{1} & rs 10877013 & 58165085 & -0.023 & $9.3 \times 10^{-3}$ & 1.57 & $7.2 \times 10^{-2}$ \\
\hline & rs 10877015 & 58167788 & -0.024 & $6.6 \times 10^{-3}$ & 1.59 & $6.2 \times 10^{-2}$ \\
\hline & rs923829 & 58174306 & -0.026 & $3.5 \times 10^{-3}$ & 1.60 & $5.9 \times 10^{-2}$ \\
\hline & rs6581155 & 58178162 & -0.024 & $6.6 \times 10^{-3}$ & 1.58 & $7.2 \times 10^{-2}$ \\
\hline \multirow{3}{*}{2} & rs 3847663 & 60542054 & 0.019 & $4.2 \times 10^{-2}$ & 0.36 & $4.8 \times 10^{-4}$ \\
\hline & rs 10784033 & 60591563 & -0.023 & $8.3 \times 10^{-3}$ & 2.48 & $7.8 \times 10^{-4}$ \\
\hline & rs 10877403 & 60605534 & 0.025 & $4.5 \times 10^{-3}$ & 0.36 & $2.0 \times 10^{-4}$ \\
\hline \multirow{3}{*}{3} & rs 7301866 & 63833069 & 0.020 & $2.9 \times 10^{-2}$ & 0.40 & $1.1 \times 10^{-3}$ \\
\hline & rs 1733676 & 63873895 & -0.012 & $2.2 \times 10^{-1}$ & 2.17 & $5.2 \times 10^{-3}$ \\
\hline & rs7133474 & 63897318 & -0.022 & $1.3 \times 10^{-2}$ & 2.32 & $2.0 \times 10^{-3}$ \\
\hline
\end{tabular}

The Bonferroni-adjusted significance level is $1.52 \times 10^{-4}(0.05 / 330)$

'Based on GRCh37s/hg19 assembly

*Regression outcomes based on imputed data using IMPUTE2 (v2.3.0) 


\section{Functional annotation of SNPs in HASs}

Given the non-coding regulatory nature associated with healthy aging, our main objective was to identify the regulatory elements and their target genes. To do so, we searched for functional SNPs that are responsible for the linkage and association outcomes. We used ChroMoS to obtain functional annotations of SNPs present in HASs. ChroMoS assigns putative functions of individual SNPs based on genetic and epigenetic data [19]. Of the SNPs examined for HAS-1, rs 10877013 was assigned strong enhancer activity in multiple cell lines (Figure 3A and 3D). Consistent with this annotation, the SNP falls within multiple transcription factor binding sites (Figure 4). For HAS-2, rs3847663 was predicted to overlap a strong enhancer element in various cell lines (Figure 3B and 3D). It falls within stretches of histone marks indicative of active regulatory elements, a DNase I hypersensitive site,

A

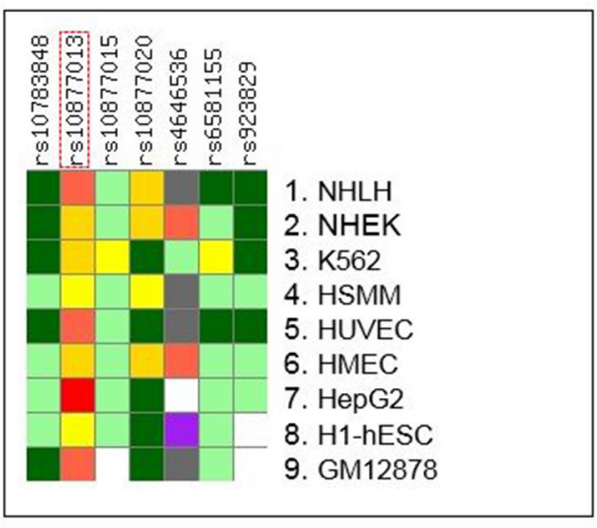

B

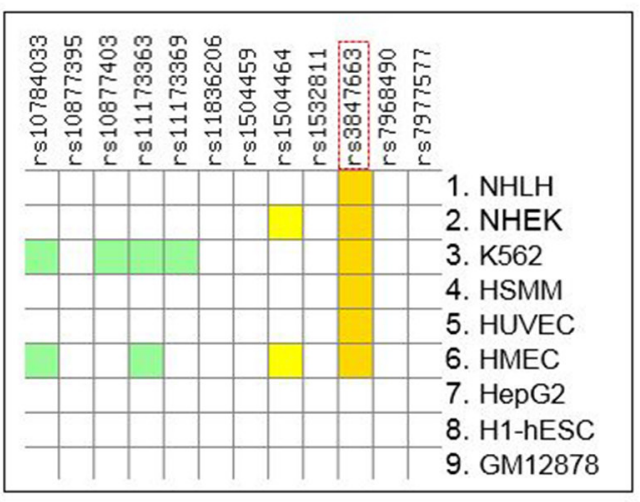

and multiple transcription factor binding sites (Figure 5). On the other hand, rs7301866 in HAS-3 was predicted to be in a Polycomb-repressed site in one cell line (Figure 3C and 3D). It is located within a segment subject to Polycomb repression (Figure 6).

\section{DISCUSSION}

According to recent database statistics compiled by LongevityMap, of the total 755 loci studied for their associations with human aging and longevity, 257 were entered as significant [20]. However, most of these genes or variants remain to be validated; only a handful of them, such as $A P O E$ and $F O X O 3 A$, have been replicated in separate studies [21]. The number of human aging studies utilizing genetic linkage analysis is much more limited, and most of these linkage results haven't been pursued

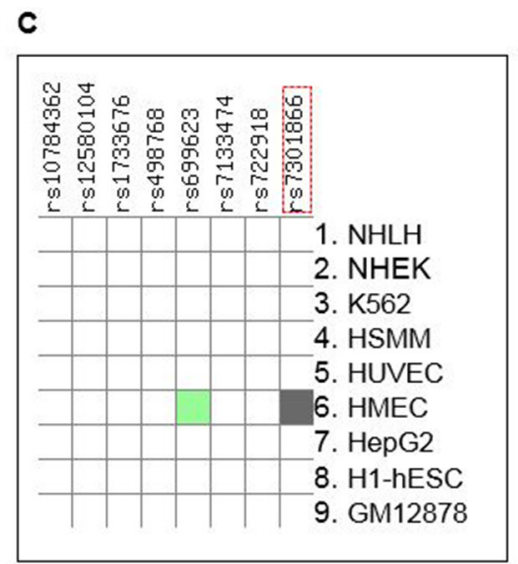

D

\begin{tabular}{|l|} 
Active promoter \\
Weak promoter \\
Inactive/poised promoter \\
Strong_enhancer \\
Strong_enhancer \\
Weak/poised enhancer \\
Weak/poised enhancer \\
Insulator \\
Transcriptional transition \\
Transcriptional elongation \\
Weak transcribed \\
Polycomb repressed \\
Heterochrom; low signal \\
Repetitive/CNW \\
Repetitive/CNW
\end{tabular}

Figure 3: Summary of results of ChroMoS (Chromatin Modified SNPs) annotation for the SNPs in HAS-1. (A), HAS2 (B), and HAS-3 (C) SNPs known or suspected to be functional are enclosed in a dotted red rectangle. (D) The genome is functionally segmented into discrete chromatin states through multivariate hidden Markov modeling of ChIP-seq data from multiple cell lines [53]. According to the 'learned' chromatin segmentation, ChroMoS graphically assigns individual noncoding SNPs to these chromatin states coded by different colors [19]. Cell lines are NHLF (normal human lung fibroblasts), NHEK (normal human epidermal keratinocytes), K562 (chronic myelogenous leukemia cells), HSMM (human skeletal muscle myoblast cells), HUVEC (human umbilical vein endothelial cells), HMEC (human mammary epithelial cells), HepG2 (human liver carcinoma cells), H1-hESC (human embryonic stem cells), and GM12878 (lymphoblastoid cells). 


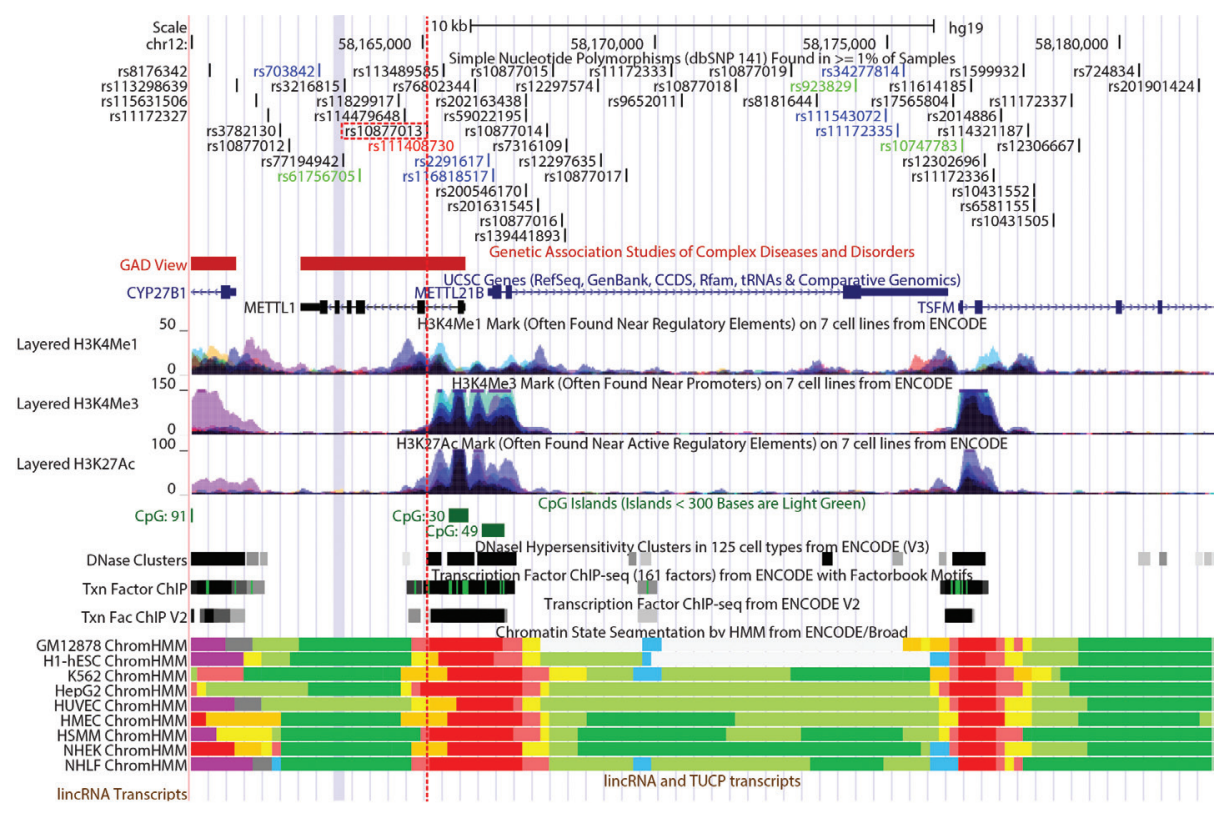

Figure 4: A close-up view of HAS-1 including rs10877013 (red-dotted line) provided by the UCSC Genome Browser [54]. SNP IDs in black are in introns, green in coding (synonymous), red in coding (non-synonymous), and blue in untranslated regions. Loci in which variants have been associated with complex diseases or disorders are shown in red blocks. The UCSC Gene track is based on gene prediction data from sources indicated. Coding exons are represented by thick blocks, non-coding or untranslated regions by relatively thin blocks, and introns by thin lines. Gene names and blocks in black represent genes entered in the Protein Data Bank (PDB) and those in blue are transcripts reviewed or validated by either the RefSeq, SwissProt or consensus coding sequence (CCDS) project. Different colors in the histone modification tracks represent results from different cell lines, and peak levels show enrichment levels of the corresponding histone marks as determined by ChIP-seq assays. The numbers following ' $\mathrm{CpG}$ ' represent $\mathrm{CpG}$ dinucleotide counts. The DNase Clusters track shows DNase hypersensitive sites with the darkness being proportional to the sensitivity. The 'Txn Factor ChIP' track shows transcription factor binding sites from ChIP-seq experiments carried out by the ENCODE project [55]. The DNA binding motifs are from the ENCODE Factorbook repository, which can be viewed as a matrix of all ENCODE transcription factor ChIP-seq datasets, arranged by cell lines [56]. The darkness is proportional to the signal strength, and the green highlights indicate the highest scoring-site motifs. The "Txn Fac ChIP V2' track is similar to the other track, but it employs a different computation method. The ChromHMM tracks, like ChroMoS, show chromatin segments corresponding to different functional states as shown in Figure 3D, according to the computational integration of ChIP-seq data from multiple cell lines using a Hidden Markov Model [57].

further. Currently, the only significant linkage peak identified in more than one study is located at $3 \mathrm{p} 24-22$ (Table 2).

By employing linkage analysis followed by association mapping, we mapped genomic sites on chromosome 12 that are linked to healthy aging, which in consequence uncovered multiple, novel genetic markers of longevity. Our approach deserves several comments. The genome-wide linkage scan is unbiased in that it doesn't need any prior knowledge. Secondly, having found a significant linkage, we were able to focus on the linkage region with a lowered power barrier. Thirdly, our phenotype (expressed as $\mathrm{FI}_{34}$ ) is well defined and characterized, with substantial familial clustering and heritability. Fourth, we leveraged the linkage analysis to establish association of gene variants with the phenotype in a separate cohort.

Typical linkage studies of human aging are carried out on the oldest-old with little consideration of the individual's health and/or function ability (Table 2). Various health deficits begin to accumulate at age 60 70, and older people accumulate deficits at different rates
$[15,22]$. Thus, elderly individuals of the same age may differ in their healthy-aging status. Because healthy aging is a significant predictor of mortality, the actual life expectancy of these individuals may substantially differ depending on their healthy-aging status. Therefore, we considered it to be more informative and productive to incorporate a validated functional measure into a linkage analysis than to base the analysis solely on chronological age. The number of studies using such a measure of healthy aging is very limited. Reed et al. [23] defined the healthy phenotype in their study based on a small number of variables: reaching age of at least 70 and the absence of medical history of several major diseases. On the other hand, similar to our study, Edwards et al. [24] incorporated a relatively large number of health variables, including variables for physical and cognitive functioning, to their phenotype of successful aging. Although statistical and genetic properties of their phenotypic measure are not known, we assume that their measure is similar to our well characterized index of healthy aging. The reason underlying this assumption is that as long as the numbers of health variables are statistically sufficient, different 


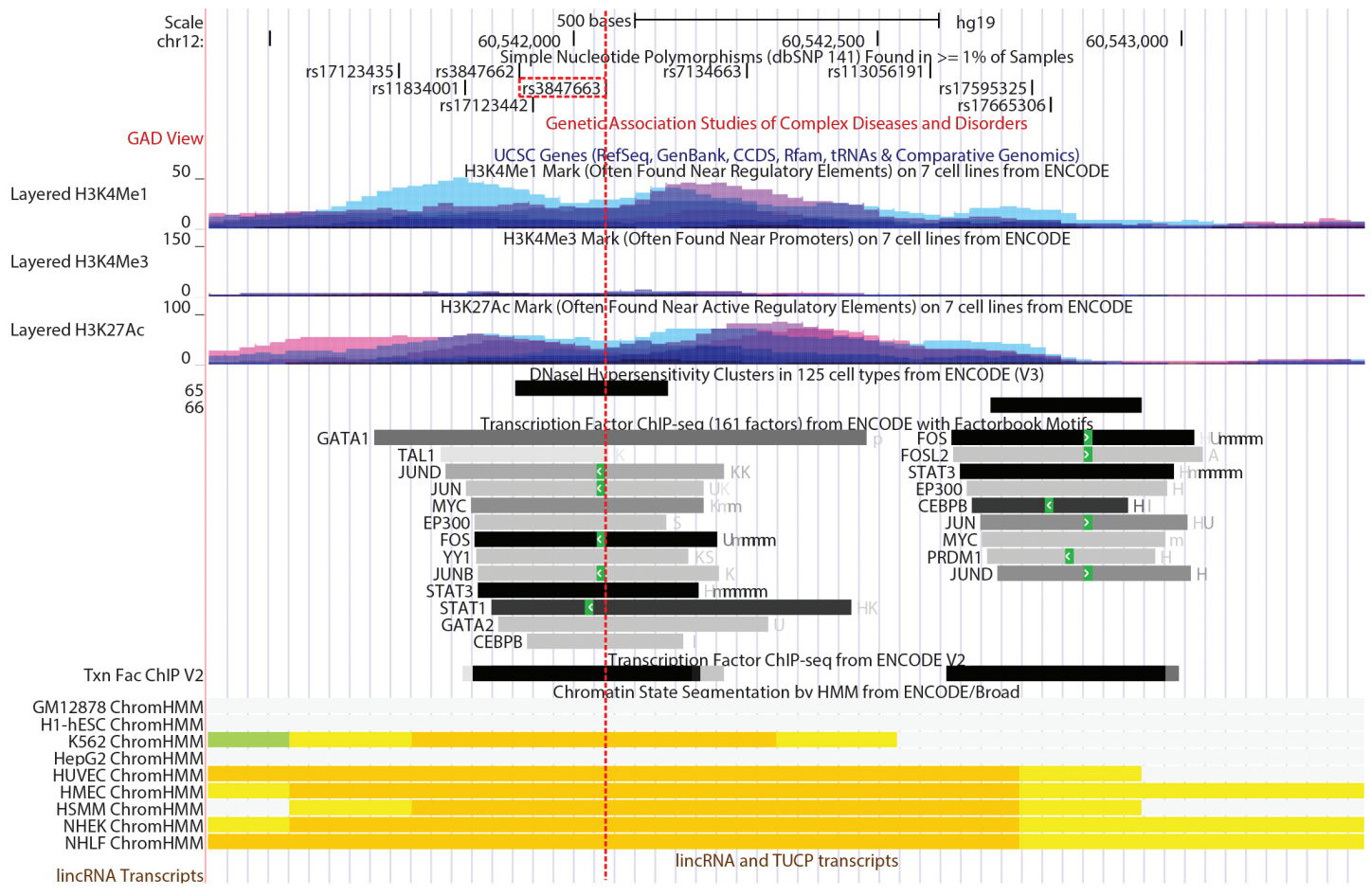

Figure 5: A close-up view of HAS-2 including rs3847663 (red-dotted line) provided by the UCSC Genome Browser. Track displays are as described in Figure 4. The names to the left of individual transcription factor binding sites are the HGNC gene names for corresponding transcription factors.

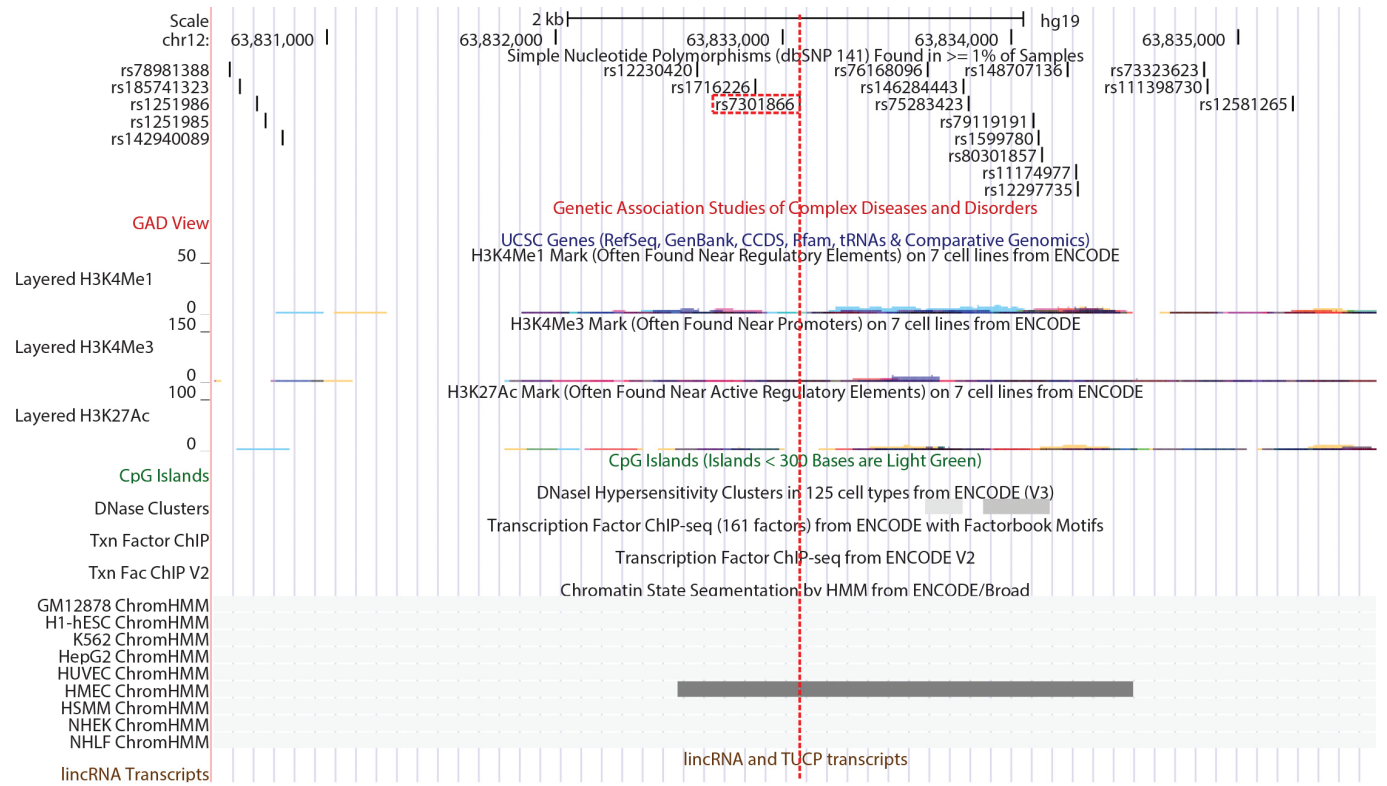

Figure 6: A close-up view of HAS-3 including rs7301866 (red-dotted line) provided by the UCSC Genome Browser. The gray-colored block in ChrommHMM tracks represents a Polycomb-repressed site. Other track displays are as described in Figure 4.

frailty indices show similar statistical properties, even if they are based on different types of health variables [25].

Few of the linkage studies have been replicated, which is common in other study designs, especially in population-based association studies [26, 27]. The linkage region at $4 \mathrm{q} 25$ was captured early on in two separate studies, but it failed to be corroborated later in another study [28]. Our study is similar to the study by Edwards et al. [24] in that both incorporated functional measures of aging. However, the linkage regions identified by the two studies differ. There could be a number of reasons for this, such as variation in study design or in genetic background. 
Table 2: Summary of linkage studies on healthy aging and longevity

\begin{tabular}{|c|c|c|c|}
\hline Phenotype & Location & LOD score ( $P$-value) & Reference \\
\hline Longevity & $4 q 25$ & $3.65\left(4.4 \times 10^{-2}\right)^{\mathrm{a}}$ & {$[58]$} \\
\hline Healthy aging & $4 q 25$ & $1.67\left(3.0 \times 10^{-3}\right)^{b}$ & {$[23]$} \\
\hline \multirow{2}{*}{ Longevity } & $3 \mathrm{p} 24.2-22.3$ & $4.02\left(3.7 \times 10^{-2}\right)^{\mathrm{c}}$ & \multirow{2}{*}[5]{} \\
\hline & $9 \mathrm{q} 31.3-34.2$ & $3.89\left(5.4 \times 10^{-2}\right)^{\mathrm{c}}$ & \\
\hline \multirow{3}{*}{ Successful aging } & Chromosome 6, & $4.49^{\mathrm{d}, \dagger}$ & \multirow{3}{*}[24]{} \\
\hline & 7 & $3.11^{\mathrm{e}}$ & \\
\hline & 14 & $4.17^{\mathrm{e}}$ & \\
\hline \multirow{5}{*}{ Successful aging } & Chromosome 6, & $3.24^{\mathrm{d}, \dagger}$ & \multirow{5}{*}[59]{} \\
\hline & 10 & $4.2^{\mathrm{e}}$ & \\
\hline & 16 & $3.3^{\mathrm{d}}$ & \\
\hline & 17 & $3.5^{\mathrm{e}}$ & \\
\hline & 20 & $3.3^{\mathrm{e}}$ & \\
\hline Longevity & $3 \mathrm{p} 24-22$ & $4.19\left(1.0 \times 10^{-5}\right)^{\mathrm{f}}$ & {$[60]$} \\
\hline \multirow{4}{*}{ Longevity } & $14 q 11.2$ & $3.47^{\mathrm{g}}$ & \multirow{4}{*}[61]{} \\
\hline & $17 q 12-22$ & $2.95^{\mathrm{g}}$ & \\
\hline & 19p13.3-p13.11 & $3.76^{g}$ & \\
\hline & 19q13.11-q13.32 & $3.57^{\mathrm{g}}$ & \\
\hline Healthy aging & $12 \mathrm{p} 13-14$ & $3.0\left(1.0 \times 10^{-4}\right)^{\mathrm{h}}$ & This study \\
\hline
\end{tabular}

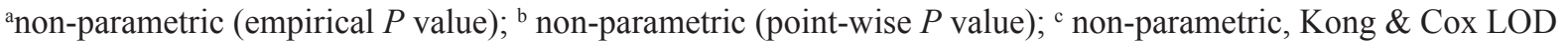
score - exponential model (empirical $P$ value); ${ }^{\mathrm{d}} \mathrm{HLOD} ;{ }^{\mathrm{e}} \mathrm{NPL}$ statistic in MERLIN; ${ }^{\mathrm{f}} \mathrm{Z}$-score (point-wise $P$ value); ${ }^{\mathrm{g}}$ non-parametric; ${ }^{\mathrm{h}}$ non-parametric, Kong \& Cox LOD score - linear model (point-wise $P$ value): ${ }^{\dagger}$ Different regions

One of the characteristics of complex traits is the presence of multiple layers of gene-gene and gene-environment interactions occurring in and between underlying genetic networks $[16,27,29]$. Frequencies of risk variants may vary across different populations, which may lead to variable gene-gene interactions [29]. Moreover, the effect of a gene can be allele- and sex-specific [30]. Consequently, it is not surprising to see different results from different studies involving different populations, where allele frequencies and environments likely differ.

In our study, we examined two separate (though related) phenotypes for the first time, healthy aging and longevity. Somewhat different outcomes were observed for association of SNPs depending on the phenotype. The allelic association tests of longevity involved both young controls and nonagenarian cases, whereas the regression tests of healthy aging involved the oldest-old cases only. Furthermore, the phenotype of healthy aging is not exactly the same as the phenotype of longevity. Although $\mathrm{FI}_{34}$ is correlated with mortality/survival, the two statistical association tests need not necessarily converge on the same SNPs. As shown earlier [15], $\mathrm{FI}_{34}$ predicts mortality better than does chronological age. Importantly, all the healthy-aging associated sites contained longevityassociated SNPs, and these results were also confirmed by permutation testing with 10,000 replicates.

All the functionally annotated SNPs that we examined in the healthy-aging associated sites (HAS) are non-coding variants. In particular, HAS-2 and -3 are located in intergenic regions barren of any known mRNA-encoding loci. Thus, our foremost task was to assign functionality to the SNPs that could be causative for the healthy-aging association. Many post genomewide association studies of human pathological traits have found non-coding variants capable of modulating gene expression by affecting transcriptional enhancer or silencer activity [31].

SNP rs10877013 in HAS-1, located within a putative binding site for the CCAAT/enhancer binding protein (C/EBP), affects enhancer activity of DNA fragments containing the SNP in an allele- and orientationdependent manner [18]. Data from long-range chromatin interaction assays indicate that the targets of this enhancer activity include a number of adjacent genes in HAS-1 [32]. Regarding HAS-2, annotation data indicate that rs3847663 overlaps an enhancer element marked by 
multiple transcription factor binding sites in the middle of a "gene desert" (Figure 5). Transcriptional enhancers are typically located a few genes away from their target genes or even on different chromosomes. Enhancers may regulate transcription of target genes through long-range interactions, mediated by the formation of chromatin loops [33]. According to the transcriptome data obtained from monocytes [34], several SNPs in HAS-2 are associated with transcription of genes on different chromosomes, with $P$ values ranging from $9.96 \times 10^{-6}$ to $1.79 \times 10^{-7}$. Although these eQTL associations are considered not significant in this particular cell type (the study-wise threshold of significance was set at $P<5.78 \times 10^{-12}$ ), these findings suggest that these healthy-aging associated sites may physically interact with other genomic sites to exert their regulatory effects over the course of healthy aging.

HAS-3 is flanked by two genes, AVPR $1 A$ and DPY19L2, whose bivalent promoters are surrounded by Polycomb-group (PcG) protein-repressed sites (Figure S4). It also contains a lincRNA-encoding locus. The human genome encodes more than 3,000 lincRNAs, and PcG complexes are often associated with lincRNAs [35]. At least some of the lincRNAs are known to mediate recruitment of the PcG complexes to target sites for transcriptional silencing [36]. Chromatin modification and compaction mediated by PcG complexes seem to spread in cis from the Polycomb binding sites, affecting nearby genes on the same chromosome [37]. Recently, Pemberton et al. [38] carried out RNA-seq and ChIP-seq on human fibroblasts and compiled a list of candidate target genes of Polycomb silencing. According to this data set combined with the ENCODE data, the best candidate genes subject to PcG silencing in HAS-2 are AVPR1A or DPY19L2 or both.

In sum, we have found a novel genomic region that is linked to healthy aging. We have taken this linkage analysis a step further, for the first time, by fine-mapping this genomic region, using a different population sample. Association mapping delineated three sites associated with healthy aging: HAS-1 and -2 seem to possess enhancer activity, whereas HAS-3 has silencer activity. HAS-1 to 3 also contain variants associated with longevity. We envision a mechanism of healthy aging and longevity based on non-coding regulatory elements as upstream regulators of multiple genes. Unraveling this mechanism and identification of the downstream target genes will significantly further our understanding of the etiology of healthy aging and longevity.

\section{METHODS}

\section{Study subjects}

The Healthy Aging Family Study (HAFS) and demographic characteristics of its participants were described elsewhere [15]. The participants are Louisiana residents who were at least 90 years old and their offspring
$(N=320)$, recruited in sibships. Ethnicity was selfdeclared. The Louisiana Healthy Aging Study (LHAS) was also described elsewhere [16]. Its participants are unrelated individuals $(N=869)$, ranging in age from 20 to over 100 years old. Ethnic affiliation was geneticallyinferred using Structure analysis (0.8 assignment probability) [16, 39]. Only European-origin participants were included in the analyses to avoid confounding by population admixture. Ages of participants were verified using both documentary evidence (birth certificates, passports, and driver's licenses) and demographic questionnaires. All participants provided informed consent according to protocols approved by the Institutional Review Boards.

\section{Genotyping}

Following genomic DNA extraction and quantification, genotypes of 5,913 biallelic SNPs for 324 subjects were generated, using the Illumina Infinium Linkage 24 set. Genotype data were imported into GenomeStudio Data Analysis Software and analyzed using the Genotyping Module. The starting dataset contained genotype data for 5, 913 SNPs from a total of 320 subjects. The project threshold (GenCall score cutoff) was set at the default value of 0.25 , and genotypes of samples with call rates $\geq 0.9$ were exported and further analyzed. SNPs not in Hardy-Weinberg proportions $(P<0.01$ using unrelated subjects) and SNPs with minor allele frequencies below $1 \%$ were eliminated using PLINK [40]. Genotyping errors were detected and removed by Mendelian error checking using PEDSTATS [41]. A genetic relationship matrix was constructed using GCTA [42]. Pedigree errors and cryptic relatedness between individuals were further investigated using PREST [43]. The likelihood for detection of pedigree errors increases with the number of genotyped markers, and the use of more than 5,000 genome-wide markers greatly facilitated unequivocal error detection. The PREST output in combination with the genetic relationship matrix and the participant enrollment table were used to correct pedigree errors. The detected errors include the presence of unrelated individuals in families, which is known to commonly occur by sample swabbing and mislabeling, misclassification of relationships within families (e.g., claimed full-sib instead of actual half-sib), and an incorrect report of ethnicity. Any genotype errors in the corrected pedigrees were removed by another application of PEDSTATS. The final dataset contains 5, 533 SNPs for 392 subjects, including 98 dummy subjects created for missing parents (only one parent was available in most of the families).

For association mapping, the Illumina GoldenGate assay was performed according to the manufacturer's instructions. SNPs were selected according to the Illumina Assay Design Tool. Following completion of the assay, all the SNPs and samples were analyzed using Illumina 
GenomeStudio, and the following quality control measures were used: sample call rates $\geq 0.95$, SNP call frequency $\geq 0.95,10 \%$ GenCall score $=0.3$, Cluster Sep $=0.1$ to exclude SNPs with overlapping genotype clusters, AB T mean $=0.2-0.8$ to exclude SNPs where the heterozygote cluster has shifted toward the homozygotes, AB R mean $>0.8$ to exclude SNPs with low intensity data.

\section{$\mathrm{FI}_{34}$ as a quantitative measure of healthy aging}

Construction of $\mathrm{FI}_{34}$ and its properties were described in detail [15]. The 34 health variables cover various diseases, symptoms, conditions, and functional abilities. They are adrenal disease, anemia, angina, asthma, bathing, body mass index, bronchitis, cataracts, chair stand, congestive heart failure, chronic obstructive pulmonary disease, diabetes, dressing, emphysema, feeding, family history of cancer, Geriatric Depression Scale, heart attack, high blood pressure (at the test), high cholesterol, history of high blood pressure, heart murmur, heart problem, kidney disease, liver disease, Mini-Mental State Exam, osteoporosis, seizure, self-rated health, semitandem balance, stroke, thyroid disease, transient ischemic attack, and urinary infection.

\section{Estimation of parental healthy aging status}

We inferred healthy aging status of each parent from offspring data as follows. In selective breeding of animals or plants, response $(\mathrm{R})$ is proportional to selection $(\mathrm{S})$ with the constant of proportionality being the narrow-sense heritability $\left(\mathrm{h}^{2}\right)[44,45]$ :

$$
\mathrm{R}=\mathrm{h}^{2} \times \mathrm{S}
$$

In other words, the average phenotypic value of offspring $\left(\mathrm{P}_{\text {off }}\right)$ is equal to $\mathrm{h}^{2}$ times the average phenotypic value of parents $\left(\mathrm{P}_{\mathrm{par}}\right)$ :

$$
\mathrm{P}_{\text {off }}=\mathrm{h}^{2} \times \mathrm{P}_{\text {par }}
$$

Narrow-sense heritability is the proportion of phenotypic variance (V) accounted for by the additive genetic variance $\left(\mathrm{V}_{\mathrm{a}}\right)$ :

$$
\mathrm{h}^{2}=\mathrm{V}_{\mathrm{a}} / \mathrm{V}
$$

With (3), equation (2) becomes

$$
\mathrm{P}_{\text {off }}=\left[\mathrm{V}_{\mathrm{a}} / \mathrm{V}\right] \times \mathrm{P}_{\mathrm{par}}
$$

$\mathrm{V}_{\mathrm{a}}$ is the covariance (Cov) between parents and offspring (slope between independent variable $\mathrm{x}$ and dependent variable y). Thus, equation (4) becomes

$$
\begin{gathered}
\mathrm{P}_{\mathrm{off}}=\left[\mathrm{Cov} / \mathrm{V}_{\mathrm{par}}\right] \times \mathrm{P}_{\mathrm{par}} \\
\left(\mathrm{V}_{\mathrm{par}}=\text { parental phenotypic variance }\right)
\end{gathered}
$$

(5) becomes

$\mathrm{Cov} / \mathrm{V}_{\mathrm{par}}$ is the regression coefficient. Thus, equation

$$
\mathrm{P}_{\text {off }}=\text { coefficient } \times \mathrm{P}_{\mathrm{par}}
$$

Thus, $\mathrm{h}^{2}$ is equal to regression coefficient (compare (2) and (6)). Rearranging (6) leads to

$$
\mathrm{P}_{\text {par }}=\mathrm{P}_{\text {off }} / \text { coefficient }
$$

Regression of offspring on only one parent underestimates narrow-sense heritability by about $50 \%$. However, our estimate of $\mathrm{h}^{2}$ of $\mathrm{FI}_{34}$ is based on intraclass correlation involving full sib pairs only [15]. Accordingly, we were able to estimate phenotypic values of parents by dividing offspring phenotypic values by $\mathrm{h}^{2}$. For each family, we took the average of offspring $\mathrm{FI}_{34}$ scores as the offspring phenotypic value and $\mathrm{h}^{2}$ as 0.39 , to infer parental $\mathrm{FI}_{34}$ at corresponding age. To use the inferred $\mathrm{FI}_{34}$ values in MERLIN analysis (see below), parents were divided into two age groups (90-94, 95-104) (Table S1), and parents in each age group were dichotomized using the lower limit of the $95 \% \mathrm{CI}$ for the mean $\mathrm{FI}_{34}$ of each age group as a cutoff, as described below.

\section{Linkage analysis}

To carry out linkage analysis, we used the $n p l$ module of MERLIN-1.1.2 with - pairs -npl command line options [46]. The -pairs option yields the Whittemore and Halpern NPL pair statistic to test for allele sharing among pairs of affected individuals, whereas the -npl option gives the Whittemore and Halpern NPL all statistic to test for allele sharing among all affected individuals [46, 47]. In general NPL all statistics were better than the pair statistics (i.e., higher LOD scores and lower $P$ values), and the linkage statistics and plots presented in this paper are from the all statistics. The $n p l$ module of MERLIN requires binary traits. The HAFS subjects were divided into four age groups: three offspring age groups (60 to 64, 65 to 69 , and 70 to 74 ) and two parent age groups (90-94, 95-104) (Table S1). The division into age groups accounts for the increase in $\mathrm{FI}_{34}$ with age, in the analyses. Offspring in each age group were dichotomized using the lower limit of the $95 \% \mathrm{CI}$ for the mean $\mathrm{FI}_{34}$ of each age group as a cutoff. The reason for using the lower limit instead of the upper limit or the mean was to be more stringent in forming the 'healthy' aging group against the 'unhealthy' aging group. If an $\mathrm{FI}_{34}$ score is lower than the cutoff, the subject is coded ' 2 ' (yes) for healthy aging; otherwise, the subject is coded ' 1 ' (no). We obtained similar results from using the lower limit of the 90\% CI (Figure S5A). With the mean $\mathrm{FI}_{34}$ value as the cutoff in each age group, we obtained the same linkage peak, but the LOD score was lower (Figure S5B). The linkage peak at 12q13-14 was not observed in all the $n p l$ runs when age group 0 (ages $<60$ ) was included (Figure S5C-E).

\section{Modeling marker-marker LD}

The LD modeling in MERLIN is based on haplotype frequency estimation within clusters of markers [48, 49]. We used -rsq 0.16 (or 0.40) along with -grid 2 (or 5) options (Figure S1). The rsq option is to create clusters of adjacent 
markers for which pairwise $r^{2}$ exceeds 0.16 (or 0.4 ), and the grid option is to have MERLIN carry out analysis at a 2-cM (or 5-cM) interval along the chromosome. Boyden and Kunkel [50] and Edwards et al. [24] used the $\mathrm{r}^{2}$ value of 0.16 .

\section{Association mapping and Manhattan plots}

We conducted a case-control association analysis using the LHAS sample. Following quality control measures as described above, we obtained genotype data for 453 SNPs in 312 subjects (136 controls of age from 31 to 59 plus 176 cases of age $\geq 90$ ). The 176 cases were divided into two age groups (90 to 94 and 95 to 103), and the cutoff $\mathrm{FI}_{34}$ value for each age group was calculated in the same way as the cutoff values for the $n p l$ linkage analysis (Table S2). The same binary coding used in the linkage analysis was used: If a $\mathrm{FI}_{34}$ score was lower than the cutoff, the subject was coded '2' (yes) for healthy aging; otherwise, the subject was coded ' 1 ' (no). All statistical analyses to test SNP associations were performed in PLINK. The additive mode of inheritance for each SNP assumed an increasing effect of its genotype with the increasing dose of the minor allele. The linear regression of raw $\mathrm{FI}_{34}$ scores on additive effects of SNPs was performed by issuing -linear command line option along with -covar and-sex options, which were to adjust for age (in a separate covariate file) and sex. Similarly, the logistic regression of dichotomized $\mathrm{FI}_{34}$ on additive effects of SNPs was carried out by using - logistic. The association for longevity between cases and controls was carried out by using the standard -assoc option, which generated asymptotic $P$ values based on 1-df $\chi^{2}$ statistics. We also performed Fisher's Exact test using -fisher option and permutation by -perm option. The PLINK outputs were directly fed into Haploview (v4.2) [51] to construct Manhattan plots.

\section{Genotype imputation}

IMPUTE2 (v2.3.0) [52] was used to impute genotypes for SNPs that were not included in the genotyping assay. Before using IMPUTE2, PLINK input files were prepared for SNPs that were located adjacent to the un-typed SNPs whose genotypes were to be imputed. The PLINK files were converted to the IMPUTE2 file format using GTOOL (v0.7.5) (http://www.well.ox.ac.uk). Imputation was carried out as directed by the user guide, using reference files downloaded from https://mathgen.stats. ox.ac.uk/impute/impute_v2.html\#reference. The output files were converted to PLINK files for association tests.

\section{ACKNOWLEDGMENTS}

This study was supported by grants from the National Institute on Aging of the National Institutes of Health (K01AG027905 to SK and P01AG022064 to SMJ), the National Institute of General Medical Sciences of the National Institutes of Health (P20GM103629) to S.M.J. and S.K., the Louisiana Board of Regents through the Millennium Trust Health Excellence Fund [HEF(2001-06)-02] to SMJ, and by the Louisiana Board of Regents RC/EEP Fund through the Tulane-LSU CTRC at LSU Interim University Hospital. We thank the CTRC for nursing services, subject testing, and blood draw, and the core lab support for blood sample processing. Preparation of genomic DNA, genotyping, and data analysis were aided by the Genomics and Biostatistics Core of the COBRE center (P20GM103629). We also thank the people of Louisiana for participation in our studies. The corresponding authors had full access to all of the data in the study and have taken responsibility for the integrity of the data and the accuracy of these data analyses.

\section{Author contributions}

S.M.J., S.K., D.A.W., and K.E.C. designed the research; S.K. and J.W. acquired data; S.K., S.M.J., and L.M. analyzed and interpreted data; and S.K. and S.M.J. prepared the manuscript.

\section{REFERENCES}

1. Finch CE, (1990). Longevity, senescence, and the genome. (Chicago: University of Chicago Press) 1990.

2. Brunet A, Berger SL. Epigenetics of aging and agingrelated disease. J Gerontol A Biol Sci Med Sci. 2014; 69:S17-20.

3. Jazwinski SM. Longevity, genes, and aging. Science. 1996; 273:54-59.

4. Guarente L, Kenyon C. Genetic pathways that regulate ageing in model organisms. Nature. 2000; 408:255-262.

5. Oeppen J, Vaupel JW. Demography. Broken limits to life expectancy. Science. 2002; 296:1029-1031.

6. Herskind AM, McGue M, Holm NV, Sorensen TI, Harvald B, Vaupel JW. The heritability of human longevity: a population-based study of 2872 Danish twin pairs born 1870-1900. Hum Genet. 1996; 97:319-323.

7. Mitchell BD, Hsueh WC, King TM, Pollin TI, Sorkin J, Agarwala R, Schaffer AA, Shuldiner AR. Heritability of life span in the Old Order Amish. Am J Med Genet. 2001; 102:346-352.

8. Perls TT, Bubrick E, Wager CG, Vijg J, Kruglyak L. Siblings of centenarians live longer. Lancet. 1998; 351:1560.

9. Perls TT, Wilmoth J, Levenson R, Drinkwater M, Cohen M, Bogan H, Joyce E, Brewster S, Kunkel L, Puca A. Life-long sustained mortality advantage of siblings of centenarians. Proc Natl Acad Sci U S A. 2002; 99:8442-8447.

10. Lander ES, Schork NJ. Genetic dissection of complex traits. Science. 1994; 265:2037-2048.

11. Sawcer S. Bayes factors in complex genetics. Eur J Hum Genet. 2010; 18:746-750. 
12. Sham PC, Purcell SM. Statistical power and significance testing in large-scale genetic studies. Nat Rev Genet. 2014; 15:335-346.

13. Li N, Shi J, Wang X, Liu G, Wang H. A combined linkage and regional association mapping validation and fine mapping of two major pleiotropic QTLs for seed weight and silique length in rapeseed (Brassica napus L.). BMC plant biology. 2014; 14:114.

14. Motte H, Vercauteren A, Depuydt S, Landschoot S, Geelen D, Werbrouck S, Goormachtig S, Vuylsteke M, Vereecke D. Combining linkage and association mapping identifies RECEPTOR-LIKE PROTEIN KINASE1 as an essential Arabidopsis shoot regeneration gene. Proc Natl Acad Sci U S A. 2014; 111:8305-8310.

15. Kim S, Welsh DA, Cherry KE, Myers L, Jazwinski SM. Association of healthy aging with parental longevity. Age (Dordr). 2013; 35:1975-1982.

16. Jazwinski SM, Kim S, Dai J, Li L, Bi X, Jiang JC, Arnold J ,Batzer MA, Walker JA, Welsh DA, Lefante CM, Volaufova J, Myers L, et al. HRAS1 and LASS1 with APOE are associated with human longevity and healthy aging. Aging Cell. 2010; 9:698-708.

17. Kim S, Welsh DA, Ravussin E, Welsch MA, Cherry KE, Myers L, Jazwinski SM. An elevation of resting metabolic rate with declining health in nonagenarians may be associated with decreased muscle mass and function in women and men, respectively. J Gerontol A Biol Sci Med Sci. 2014; 69:650-656.

18. Alcina A, Fedetz M, Fernandez O, Saiz A, Izquierdo G, Lucas M, Leyva L, Garcia-Leon JA, Abad-Grau Mdel M, Alloza I, Antiguedad A, Garcia-Barcina MJ, Vandenbroeck $\mathrm{K}$, et al. Identification of a functional variant in the KIF5A-CYP27B1-METTL1-FAM119B locus associated with multiple sclerosis. J Med Genet. 2013; 50:25-33.

19. Barenboim M, Manke T. ChroMoS: an integrated web tool for SNP classification, prioritization and functional interpretation. Bioinformatics. 2013; 29:2197-2198.

20. Budovsky A, Craig T, Wang J, Tacutu R, Csordas A, Lourenco J, Fraifeld VE, de Magalhaes JP. LongevityMap: a database of human genetic variants associated with longevity. Trends Genet. 2013; 29:559-560.

21. Brooks-Wilson AR. Genetics of healthy aging and longevity. Hum Genet. 2013; 132:1323-1338.

22. Mitnitski A, Bao L, Rockwood K. Going from bad to worse: a stochastic model of transitions in deficit accumulation, in relation to mortality. Mech Ageing Dev. 2006; 127:490-493.

23. Reed T, Dick DM, Uniacke SK, Foroud T, Nichols WC. Genome-wide scan for a healthy aging phenotype provides support for a locus near D4S1564 promoting healthy aging. J Gerontol A Biol Sci Med Sci. 2004; 59:227-232.

24. Edwards DR, Gilbert JR, Jiang L, Gallins PJ, Caywood L, Creason M, Fuzzell D, Knebusch C, Jackson CE,
Pericak-Vance MA, Haines JL, Scott WK. Successful aging shows linkage to chromosomes 6, 7, and 14 in the Amish. Ann Hum Genet. 2011; 75:516-528.

25. Rockwood K, Mitnitski A. Frailty in relation to the accumulation of deficits. J Gerontol A Biol Sci Med Sci. 2007; 62:722-727.

26. Buchanan AV, Weiss KM, Fullerton SM. Dissecting complex disease: the quest for the Philosopher's Stone? Int J Epidemiol. 2006; 35:562-571.

27. Hirschhorn JN, Lohmueller K, Byrne E, Hirschhorn K. A comprehensive review of genetic association studies. Genet Med. 2002; 4:45-61.

28. Nebel A, Croucher PJ, Stiegeler R, Nikolaus S, Krawczak M, Schreiber S. No association between microsomal triglyceride transfer protein (MTP) haplotype and longevity in humans. Proc Natl Acad Sci U S A. 2005; 102:7906-7909.

29. Marchini J, Donnelly P, Cardon LR. Genome-wide strategies for detecting multiple loci that influence complex diseases. Nat Genet. 2005; 37:413-417.

30. Magwire MM, Yamamoto A, Carbone MA, Roshina NV, Symonenko AV, Pasyukova EG, Morozova TV, Mackay TF. Quantitative and molecular genetic analyses of mutations increasing Drosophila life span. PLoS Genet. 2010; 6:e1001037.

31. Edwards SL, Beesley J, French JD, Dunning AM. Beyond GWASs: illuminating the dark road from association to function. Am J Hum Genet. 2013; 93:779-797.

32. Chepelev I, Wei G, Wangsa D, Tang Q, Zhao K. Characterization of genome-wide enhancer-promoter interactions reveals co-expression of interacting genes and modes of higher order chromatin organization. Cell Res. 2012; 22:490-503.

33. Sanyal A, Lajoie BR, Jain G, Dekker J. The long-range interaction landscape of gene promoters. Nature. 2012; 489:109-113.

34. Zeller T, Wild P, Szymczak S, Rotival M, Schillert A, Castagne R, Maouche S, Germain M, Lackner K, Rossmann H, Eleftheriadis M, Sinning CR, Schnabel RB, et al. Genetics and beyond-the transcriptome of human monocytes and disease susceptibility. PLoS One. 2010; 5:e10693.

35. Khalil AM, Guttman M, Huarte M, Garber M, Raj A, Rivea Morales D, Thomas K, Presser A, Bernstein BE, van Oudenaarden A, Regev A, Lander ES, Rinn JL. Many human large intergenic noncoding RNAs associate with chromatin-modifying complexes and affect gene expression. Proc Natl Acad Sci U S A. 2009; 106:11667-11672.

36. Marin-Bejar O, Marchese FP, Athie A, Sanchez Y, Gonzalez J, Segura V, Huang L, Moreno I, Navarro A, Monzo M, Garcia-Foncillas J, Rinn JL, Guo S, et al. Pint lincRNA connects the $\mathrm{p} 53$ pathway with epigenetic silencing by the Polycomb repressive complex 2. Genome Biol. 2013; 14:R104. 
37. Di Croce L, Helin K. Transcriptional regulation by Polycomb group proteins. Nat Struct Mol Biol. 2013; 20:1147-1155.

38. Pemberton H, Anderton E, Patel H, Brookes S, Chandler H, Palermo R, Stock J, Rodriguez-Niedenfuhr M, Racek T, de Breed L, Stewart A, Matthews N, Peters G. Genome-wide colocalization of Polycomb orthologs and their effects on gene expression in human fibroblasts. Genome Biol. 2014; 15:R23.

39. Pritchard JK, Stephens M, Donnelly P. Inference of population structure using multilocus genotype data. Genetics. 2000; 155:945-959.

40. Purcell S, Neale B, Todd-Brown K, Thomas L, Ferreira MA, Bender D, Maller J, Sklar P, de Bakker PI, Daly MJ, Sham PC. PLINK: a tool set for whole-genome association and population-based linkage analyses. Am J Hum Genet. 2007; 81:559-575.

41. Wigginton JE, Abecasis GR. PEDSTATS: descriptive statistics, graphics and quality assessment for gene mapping data. Bioinformatics. 2005; 21:3445-3447.

42. Yang J, Lee SH, Goddard ME, Visscher PM. GCTA: a tool for genome-wide complex trait analysis. Am J Hum Genet. 2011; 88:76-82.

43. Sun L, Wilder K, McPeek MS. Enhanced pedigree error detection. Hum Hered. 2002; 54:99-110.

44. Akesson M, Bensch S, Hasselquist D, Tarka M, Hansson B. Estimating heritabilities and genetic correlations: comparing the 'animal model' with parent-offspring regression using data from a natural population. PLoS One. 2008; 3:e1739.

45. Visscher PM, Hill WG, Wray NR. Heritability in the genomics era-concepts and misconceptions. Nat Rev Genet. 2008; 9:255-266.

46. Abecasis GR, Cherny SS, Cookson WO, Cardon LR. Merlin-rapid analysis of dense genetic maps using sparse gene flow trees. Nat Genet. 2002; 30:97-101.

47. Whittemore AS, Halpern J. A class of tests for linkage using affected pedigree members. Biometrics. 1994; 50:118-127.

48. Abecasis GR, Wigginton JE. Handling marker-marker linkage disequilibrium: pedigree analysis with clustered markers. Am J Hum Genet. 2005; 77:754-767.

49. Levinson DF, Holmans P. The effect of linkage disequilibrium on linkage analysis of incomplete pedigrees. BMC Genet. 2005; 6:S6.

50. Boyden SE, Kunkel LM. High-density genomewide linkage analysis of exceptional human longevity identifies multiple novel loci. PLoS One. 2010; 5:e12432.
51. Barrett JC, Fry B, Maller J, Daly MJ. Haploview: analysis and visualization of LD and haplotype maps. Bioinformatics. 2005; 21:263-265.

52. Howie B, Fuchsberger C, Stephens M, Marchini J, Abecasis GR. Fast and accurate genotype imputation in genome-wide association studies through pre-phasing. Nat Genet. 2012; 44:955-959.

53. Ernst J, Kheradpour P, Mikkelsen TS, Shoresh N, Ward LD, Epstein CB, Zhang X, Wang L, Issner R, Coyne M, $\mathrm{Ku}$ M, Durham T, Kellis M, et al. Mapping and analysis of chromatin state dynamics in nine human cell types. Nature. 2011; 473:43-49.

54. Kent WJ, Sugnet CW, Furey TS, Roskin KM, Pringle TH, Zahler AM, Haussler D. The human genome browser at UCSC. Genome Res. 2002; 12:996-1006.

55. Rosenbloom KR, Dreszer TR, Pheasant M, Barber GP, Meyer LR, Pohl A, Raney BJ, Wang T, Hinrichs AS, Zweig AS, Fujita PA, Learned K, Rhead B, et al. ENCODE wholegenome data in the UCSC Genome Browser. Nucleic Acids Res. 2010; 38:D620-625.

56. Wang J, Zhuang J, Iyer S, Lin XY, Greven MC, Kim BH, Moore J, Pierce BG, Dong X, Virgil D, Birney E, Hung JH, Weng Z. Factorbook.org: a Wiki-based database for transcription factor-binding data generated by the ENCODE consortium. Nucleic Acids Res. 2013; 41:D171-176.

57. Ernst J, Kellis M. ChromHMM: automating chromatinstate discovery and characterization. Nat Methods. 2012; 9:215-216

58. Puca AA, Daly MJ, Brewster SJ, Matise TC, Barrett J, Shea-Drinkwater M, Kang S, Joyce E, Nicoli J, Benson E, Kunkel LM, Perls T. A genome-wide scan for linkage to human exceptional longevity identifies a locus on chromosome 4. Proc Natl Acad Sci U S A. 2001; 98:10505-10508.

59. Edwards DR, Gilbert JR, Hicks JE, Myers JL, Jiang L, Cummings AC, Guo S, Gallins PJ, Konidari I, Caywood L, Reinhart-Mercer L, Fuzzell D, Knebusch C, et al. Linkage and association of successful aging to the $6 \mathrm{q} 25$ region in large Amish kindreds. Age (Dordr). 2013; 35:1467-1477.

60. Kerber RA, O'Brien E, Smith KR, Cawthon RM. Familial excess longevity in Utah genealogies. J Gerontol A Biol Sci Med Sci. 2001; 56:B130-139.

61. Beekman M, Blanche H, Perola M, Hervonen A, Bezrukov V, Sikora E, Flachsbart F, Christiansen L, De Craen AJ, Kirkwood TB, Rea IM, Poulain M, Robine JM, et al. Genome-wide linkage analysis for human longevity: Genetics of Healthy Aging Study. Aging Cell. 2013; 12:184-193. 